\title{
Efficacy and safety of Jianzhong decoction in treating peptic ulcers: a meta-analysis of 58 randomised controlled trials with 5192 patients
}

Yan Sun ${ }^{1 *}$, Jinping Zhang ${ }^{1}$ and Yuanyuan Chen $^{2}$

\begin{abstract}
Background: Jianzhong decoction is widely used to treat peptic ulcers; however, due to lack of systematic evaluations, its clinical efficacy remains controversial. We performed meta-analysis to evaluate the efficacy and safety of Jianzhong decoction in treating peptic ulcers.

Methods: Studies were systematically retrieved from PubMed, Embase, Cochrane library, China National Knowledge Infrastructure, Wanfang Database, Chongqing VIP, China Biology Medicine disc (CBMdisc), and references cited in related studies/reviews. Extracted data included the total effective rate, helicobacter pylori eradication rates, recurrence rate, and adverse reaction rate. Fifty-eight randomised controlled trials involving 5192 patients were included in the final analysis.

Results: Results showed that Jianzhong decoction therapy was more effective than conventional Western medicine therapy (total effective rate, odds ratio $[\mathrm{OR}]=4.29,95 \%$ confidence interval $[\mathrm{Cl}]$ : 3.51-5.23, $P=0.000$; helicobacter pylori eradication rates, $\mathrm{OR}=2.10,95 \% \mathrm{Cl}: 1.69-2.61, P=0.000$; recurrence rate, $\mathrm{OR}=0.23,95 \% \mathrm{Cl}: 0.18-0.29, P=0$. 000; and adverse reaction rate, $\mathrm{OR}=0.20,95 \% \mathrm{Cl}: 0.12-0.33, P=0.000$ ).

Conclusions: Jianzhong decoction increased the total effective rate and helicobacter pylori eradication rate, and lowered the recurrence and adverse reaction rates. The results of this study can be used as a guide for clinical treatment of peptic ulcers.
\end{abstract}

Keywords: Jianzhong decoction, Peptic ulcer, Randomised controlled trials, Meta-analysis

\section{Background}

Peptic ulcer (PU) refers to the ulcer in the gastrointestinal (GI) mucosa occurring due to the erosion induced by gastric acid and pepsin. Gastric and duodenal ulcers are the most commonly seen PUs in clinical practice. Typically, PU has the unique characteristics of selfhealing and recurrence, as well as a relatively high incidence rate with up to a $50 \%$ short-term incidence rate [1]. Moreover, PU has a complicated pathogenesis, including excessive gastric acid secretion, the necessitation of non-steroidal anti-inflammatory drugs (NSAIDs),

\footnotetext{
* Correspondence: nkyysy@sina.com

'Department of Chinese Medicine, Nankai Hospital, No. 6 Changjiang Road, Nankai District, Tianjin 300100, China

Full list of author information is available at the end of the article
}

Helicobacter pylori $(\mathrm{Hp})$ infection, and gastric mucosal injury [2]. Currently, PU is mainly treated with the triple or quadruple therapy of Western medicine (WM), which eradicates $\mathrm{Hp}$ and protects the gastric mucosa by inhibiting gastric acid secretion. Patients treated with WM show significant symptomatic improvements, but have a relatively higher short-term recurrence rate and a poor long-term therapeutic efficacy. Additionally, patients become susceptible to adverse reactions during WM treatment $[2,3]$. Traditional Chinese medicine (TCM) and integrated medicine are the current focus of research addressing the PU treatment. In the TCM theory, middlejiao (the middle part of a human being) is a key location for human digestion. And many digestive painful diseases can be treated by Jianzhong decoction. The name, 
Jianzhong, means construct the qi of middle-jiao. Clinically, TCM uses some combinations of sweet and warmnatured herbs as Jianzhong decoction. Here, the main herbs in the decoction are Ginger, Cassia twig, Radix paeoniae alba, Jujube and Licorice. Previous studies have confirmed that Jianzhong decoction has been widely applied in the clinical treatment of PU using various prescriptions; however, their therapeutic efficacies are still controversial [4]. Several studies have shown that Jianzhong decoction outperforms the conventional WM in terms of therapeutic efficacy against PU with a high healing rate, low recurrence rate, high $\mathrm{Hp}$ eradication rate, and a significantly reduced ulcer area. However, other studies showed that Jianzhong decoction has no significant advantage over the conventional WM in treating PU with respect to the total effective rate $[5,6]$. Therefore, our study is aimed at gathering randomised controlled trials (RCTs) that involved Jianzhong decoction in PU treatment, in order to systematically evaluate its therapeutic efficacy and safety and provide the basis for clinical PU treatment.

\section{Methods}

\section{Literature search}

PubMed, Embase, Cochrane library, China National Knowledge Infrastructure (CNKI), Wanfang Database, China Science and Technology (CSTJ) Database (Chongqing VIP), and China Biology Medicine disc (CBMdisc) were subjected to computational searches up until November 16, 2016. The Chinese search keywords were Jianzhong decoction, PU, gastric ulcer, duodenal ulcer, ulcerative colitis, and Crohn's disease. The English search keywords were Jianzhong decoction, Jianzhong tang, Jianzhong, peptic ulcer, gastric ulcer, duodenal ulcers, digestibility ulcer, ulcer, peptic colitis, Crohn's disease, and clinical. In addition, we further searched the references cited in relevant literature reviews in order to avoid any oversights.

\section{Literature inclusion and exclusion criteria}

Inclusion criteria were: (1) Research Type: Randomized Controlled Trials (RCTs); (2) patients in the treatment group were treated with mainly Jianzhong decoction or Jianzhong decoction integrated with WM and/or other treatment(s); while patients in the control group were treated with placebo or conventional WM; and (3) the outcome indicators included at least one of the following: total effective rate, $\mathrm{Hp}$ eradication rate, recurrence rate, and/or adverse reactions.

Exclusion criteria were: (1) irrelevant studies; (2) animal studies; (3) literature reviews; (4) case and expert reports; (5) non-RCTs; (6) studies that did not use Jianzhong decoction in treating PU; (7) studies related to GI bleeding, pyloric obstruction, and other severe complications; (8) studies that used Jianzhong decoction as adjuvant therapy; (9) duplicate publications; (10) Jianzhong prescription in non- decoction; and (11) No desired outcomes (studies providing no extractable data).

\section{Literature selection and data extraction}

The titles and abstracts of the retrieved articles were read independently by two researchers. After excluding the articles that did not meet the inclusion criteria, full texts of the remaining articles were perused in order to validate their inclusion. Any disagreement regarding the inclusion of articles was solved via discussion or decided by a third researcher. Both researchers independently extracted the data from the included studies. These included the names of the authors, year of publication, study samples, interventional measures of the experimental and control groups, efficacy evaluation indicators, treatment course, type of ulcer, follow-up duration, randomisation methods, baseline equilibrium, blinded experiment, and results.

\section{Research quality evaluation}

The methodological quality of the included studies was evaluated using the modified Jadad scale [7]. The evaluated items included the random sequence generation, allocation concealment, blinded experiment, and lost to follow-up. The range of total RCT scores were 1-7, wherein, studies with scores of 1-3 were considered of low quality while studies with scores of 4-7 were considered of high quality. The evaluation of data quality was performed independently by two evaluators and any disagreement was resolved via discussion.

\section{Statistical analysis}

The meta-analysis was performed using the Stata statistical software (Version 12.0). The count data were expressed as odds ratio (OR) and 95\% confidence intervals (CIs). The $I^{2}$ index was used to describe the heterogeneity among studies; wherein $I^{2}<50 \%$ denotes the absence of heterogeneity among studies that were analysed using the fixed effects model whereas studies with $I^{2}>50 \%$ were analysed using the random effects model. Potential publication bias was analysed using the funnel plot, Egger's test, and Begg's test. The stability of the results was evaluated using the sensitivity analysis, where all included studies were excluded one by one and the remaining studies were subjected to meta-analysis again to check for any change in the results before and after the exclusion. Based on the assumption that publication bias leads to asymmetry in funnel plots, we further analysed the robustness of the results using the nonparametric trim and filling method. Statistical significance was considered at $P<0.05$. 


\section{Results}

\section{Search results and the general situation of included} studies

The workflow of the literature search and selection is illustrated in Fig. 1. A total of 1246 articles consisting of 42 English articles and 1204 Chinese articles were retrieved according to the search strategy and data collection method. A total of 114 duplicate publications were excluded. Moreover, a total of 137 articles were preliminarily included after reading the titles and abstracts. We further examined the full texts of these 137 articles and excluded nine non-PU articles, 19 duplicate publications, 13 non-Jianzhong decoction articles, five non-RCTs, 16 case reports, six literature reviews, three articles with no extractable data, two conference papers, two articles with inconsistent control drugs, and one article reporting GI bleeding, pyloric obstruction, and other complications. We finally included a total of 58 articles, all of which were published in China [5, 6, 8-63]. The basic characteristics of these included studies are shown in Table 1. Briefly, these 58 studies were divided into a number of categories. Twenty-one studies used Huangqi Jianzhong decoction or Huangqi Jianzhong decoction combined with other prescriptions or therapies and WM [13-15, 18, 20, 23, 31, 34, 37, 38, 40, 42, 43, 48, $49,51-54,58,60,63]$. Sixteen studies used Xiaojianzhong decoction alone or Xiaojianzhong decoction combined with other prescriptions and WM [5, 6, 8, $9,12,17,19,21,25,27,28,30,35,36,39,41]$. Eleven studies used Huangqi Jianzhong decoction alone or Huangqi Jianzhong decoction combined with other prescriptions $[24,32,45,46,50,55,57,59,61$, 62]. Nine studies used Xiaojianzhong decoction alone or Xiaojianzhong decoction combined with other prescriptions [10, 11, 16, 22, 26, 29, 33, 44, 55]. A total of 33 studies clearly stated the type of PU studied, which mainly included gastric ulcer, duodenal ulcer, or the combination of both $[9,15,18,20,24,27,31-39,41,42,45$, 47-50, 52, 53, 55-63], while other studies did not clearly state the type of the ulcer. The included studies recruited a total of 5192 patients, consisting of 2801 patients from the treatment group and 2391 patients from the control group.

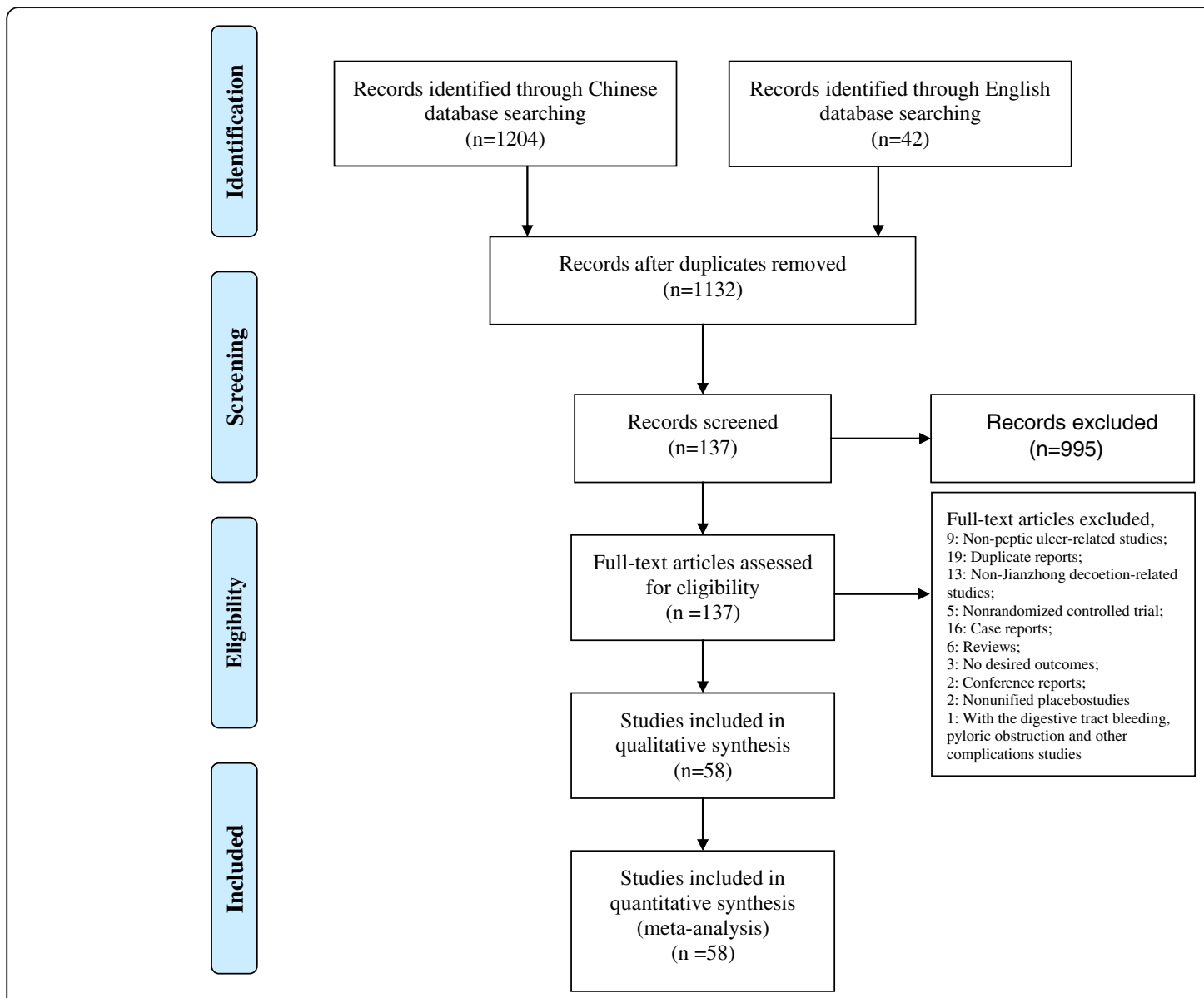

Fig. 1 PRISMA 2009 Flow Diagram 


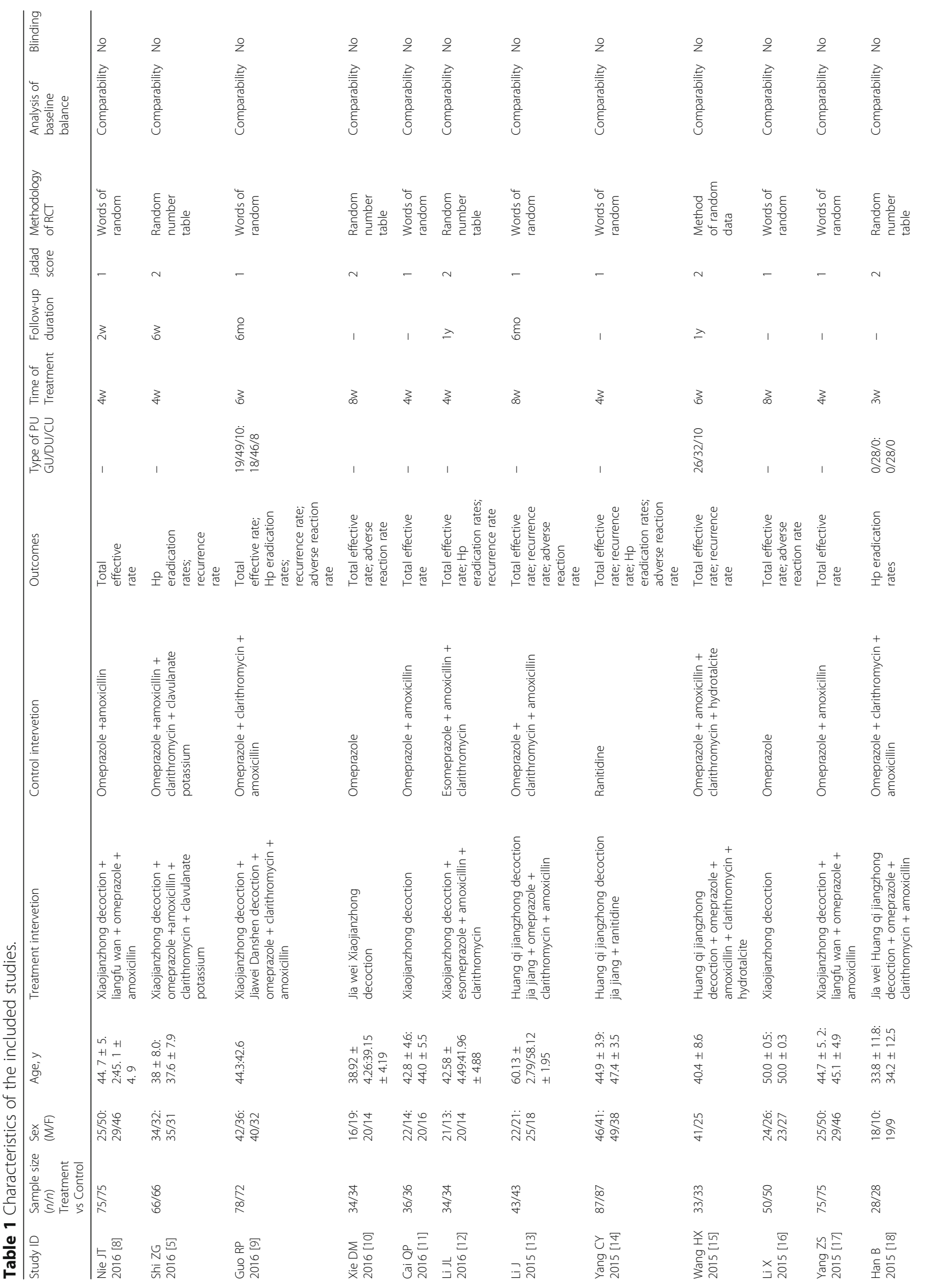




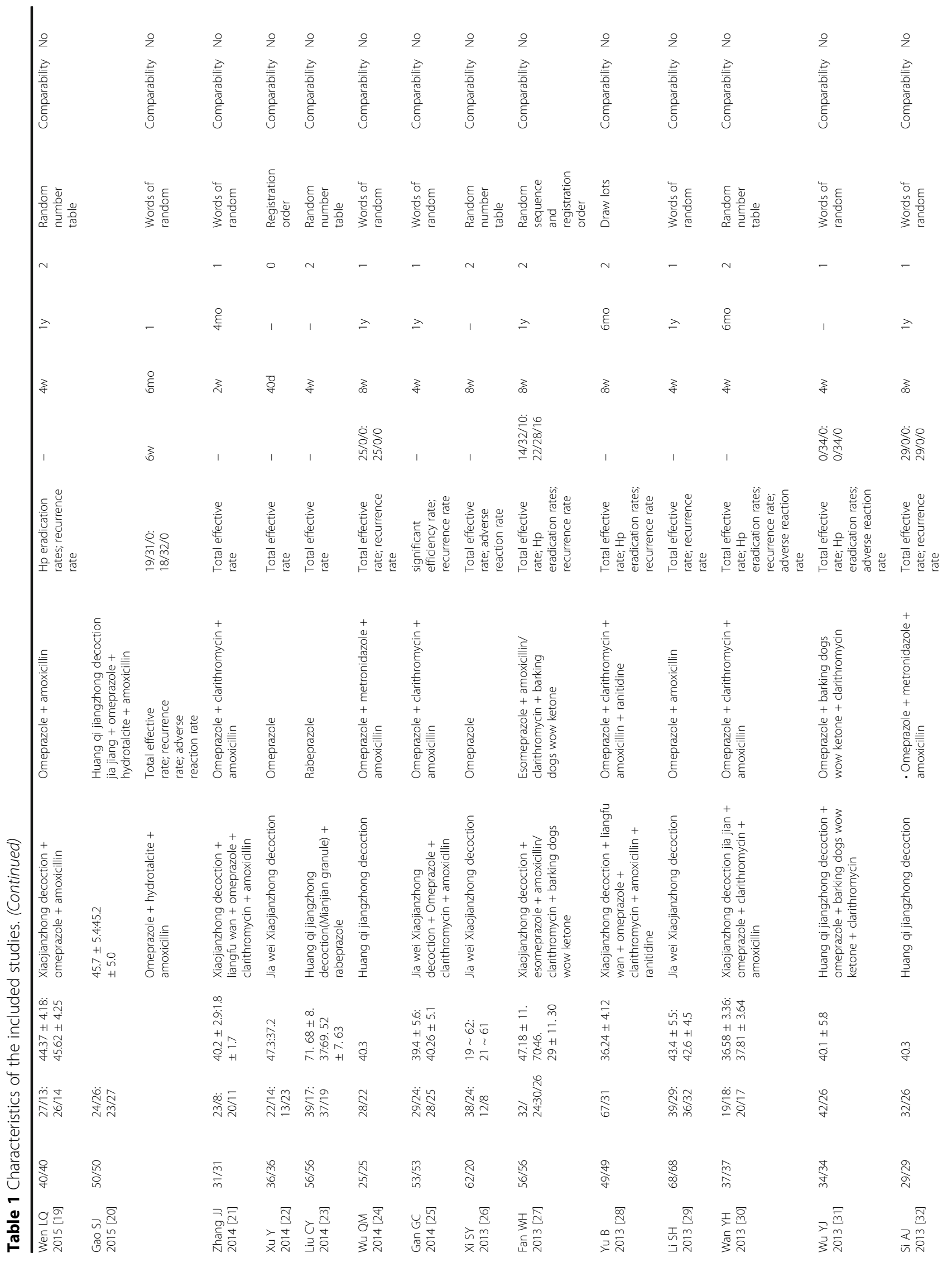




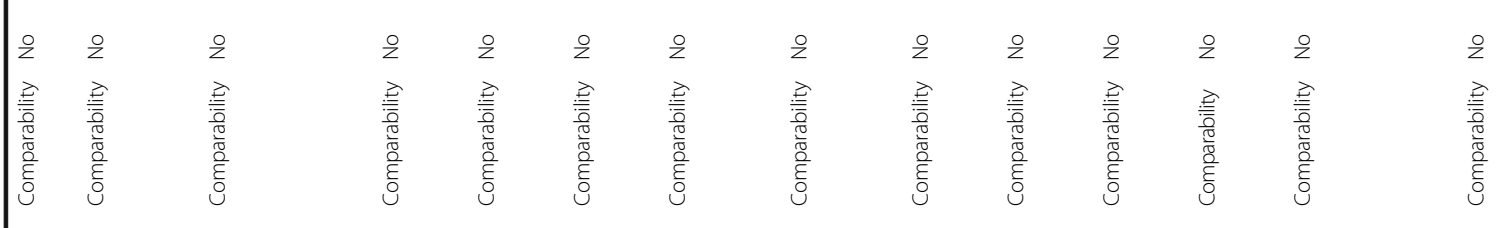

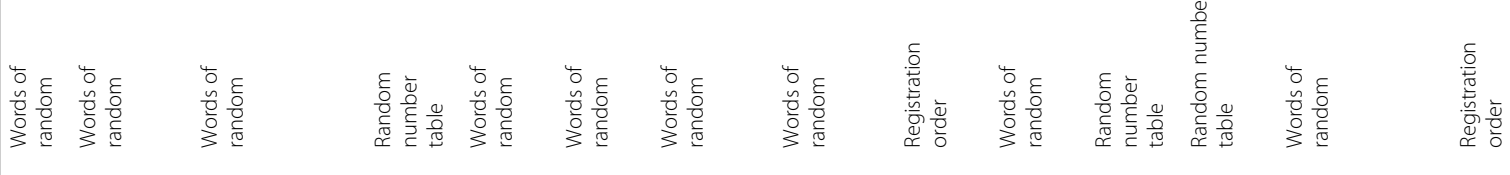

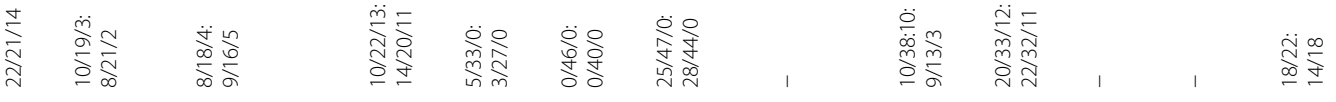

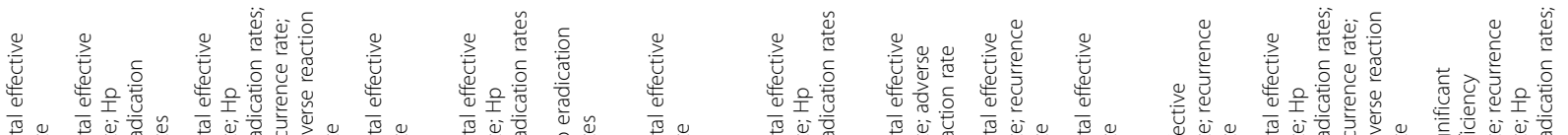

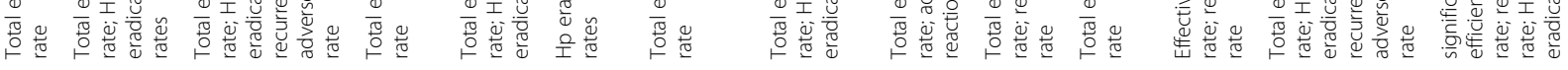

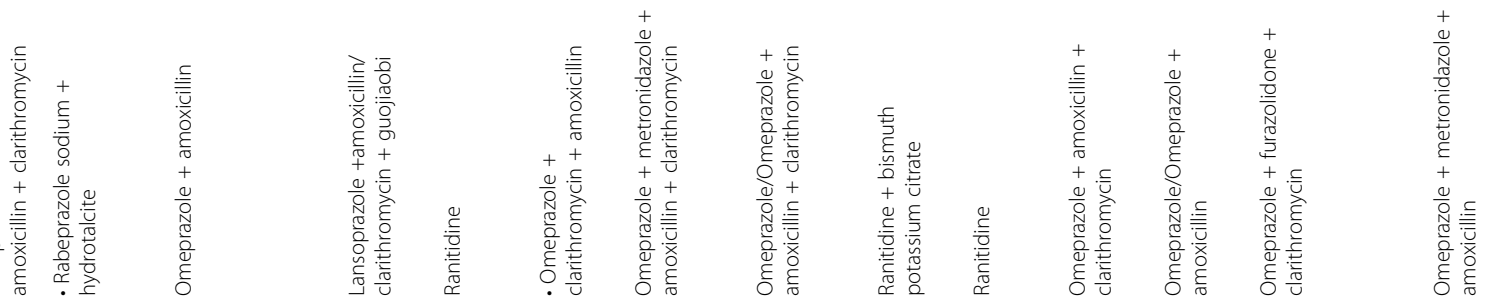

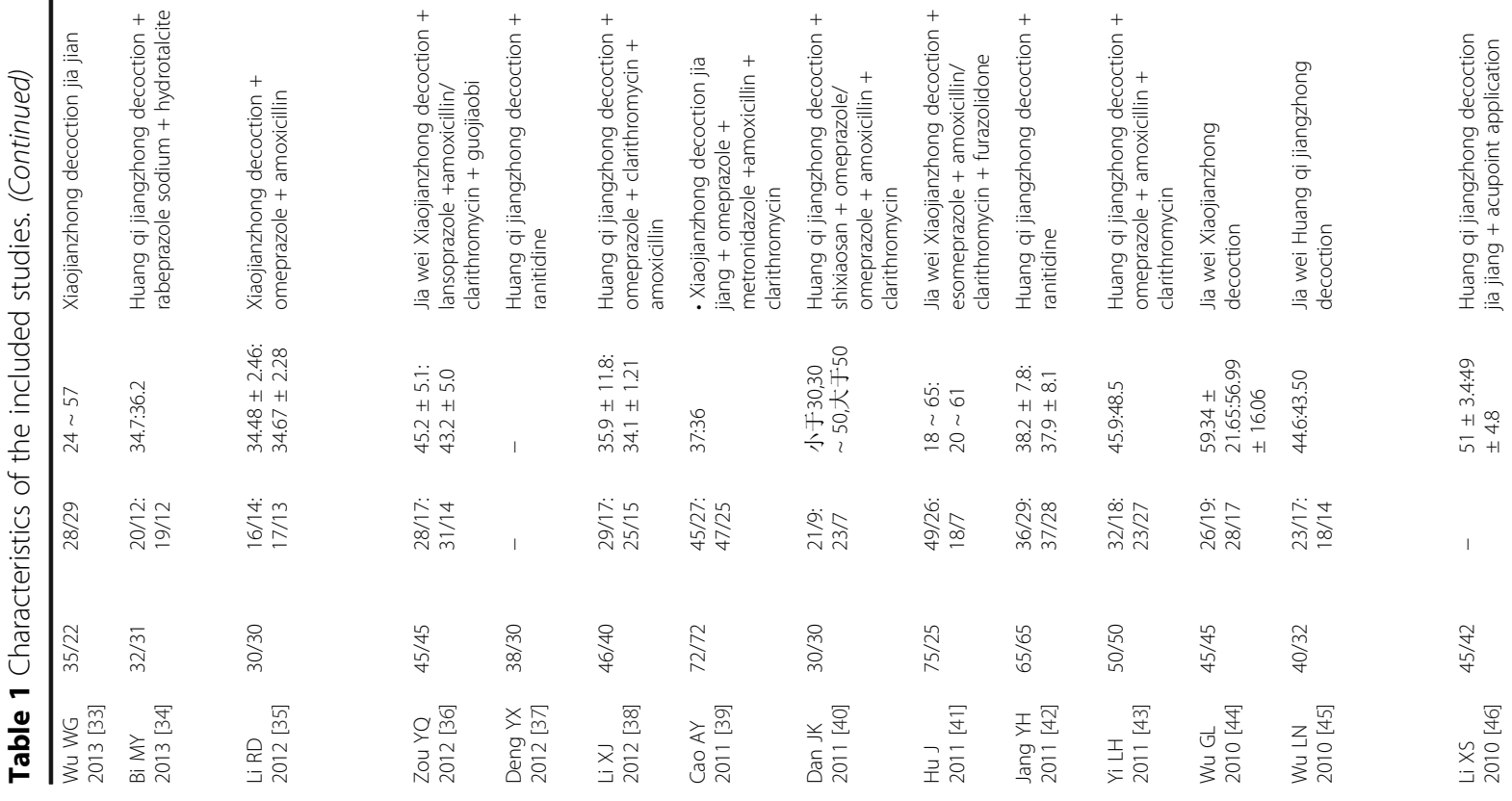




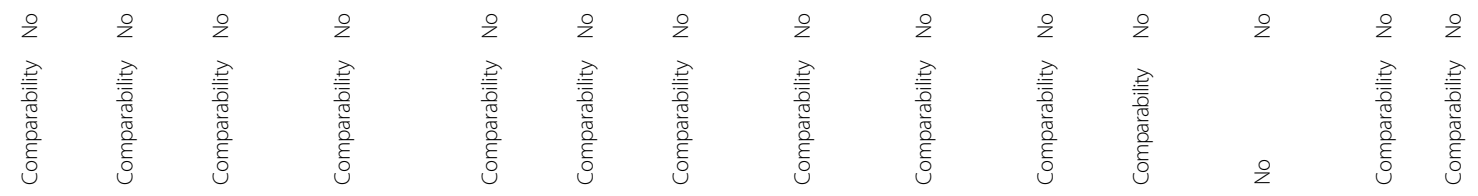

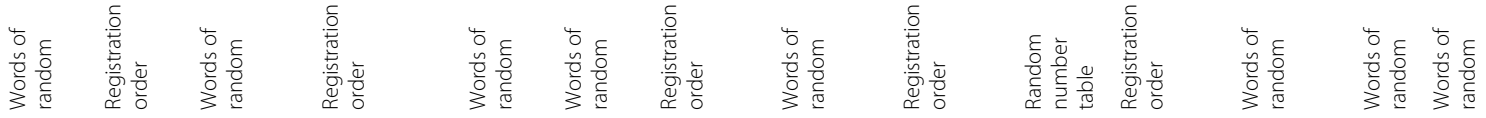
$\begin{array}{llllllllll}3 & 3 & 3 & 3 & 3 & 3 & 3 & 3 & 3 & 3\end{array}$

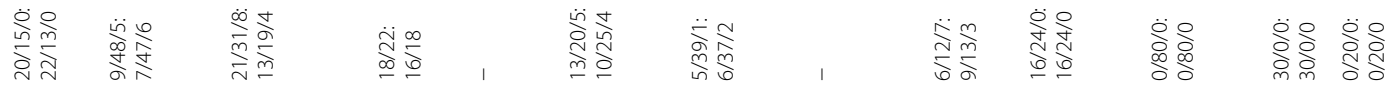

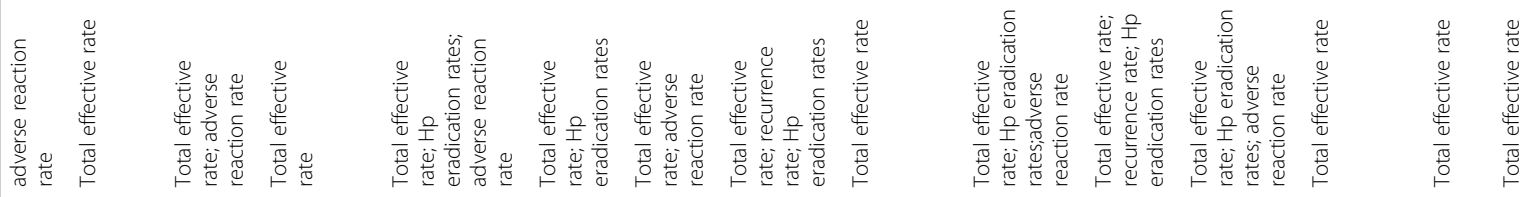
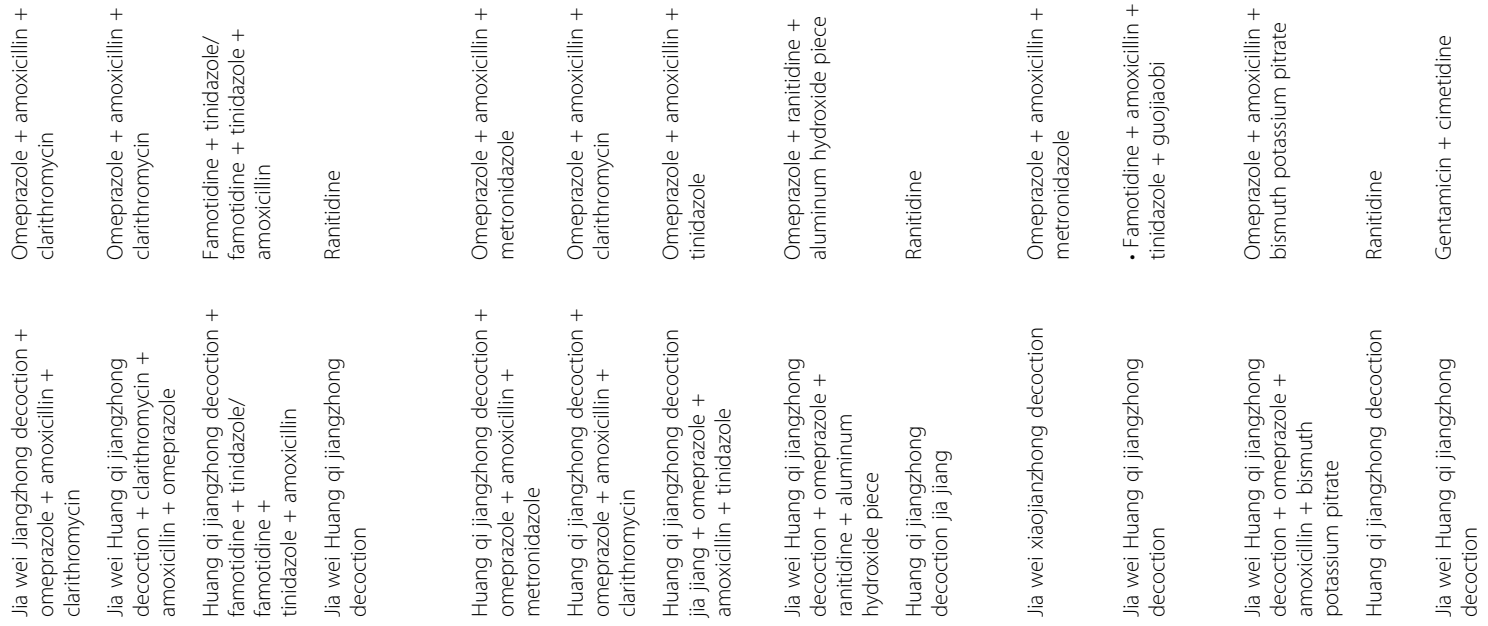

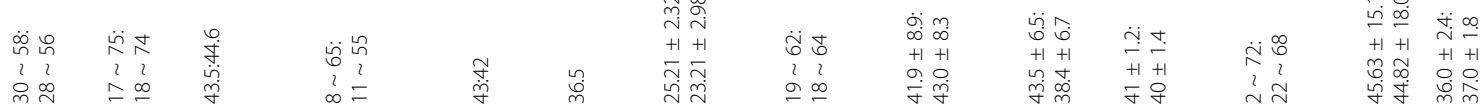

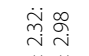

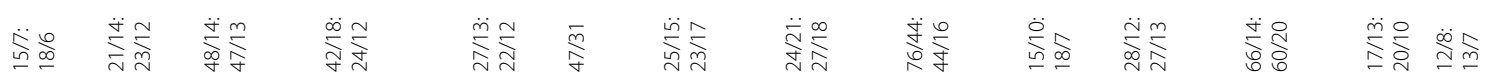

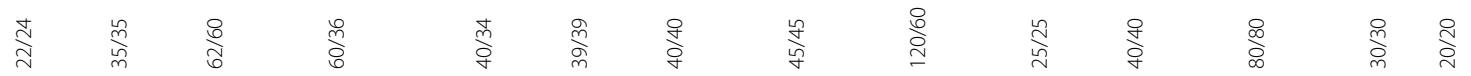

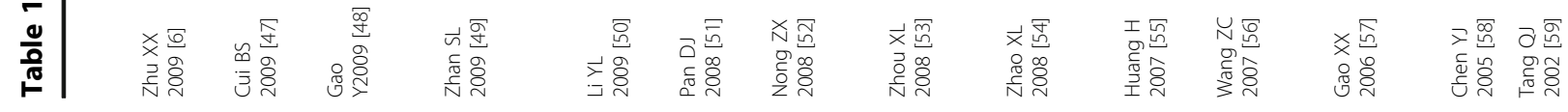




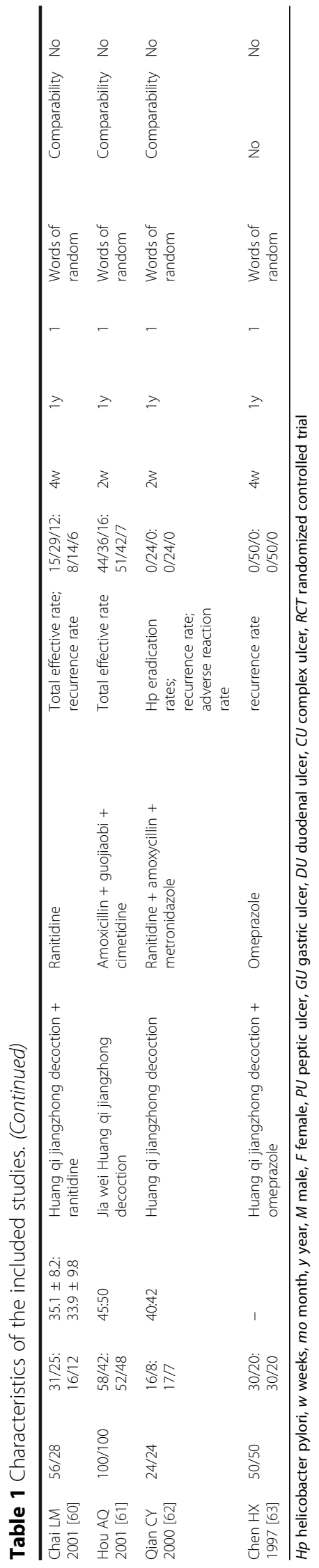




\section{Evaluation of the methodological quality of included studies}

Among those 58 studies, two studies did not specify the baseline equilibrium [57, 63], while the remaining 56 studies mentioned that the treatment and control groups were "comparable". There were 24 studies that did not clearly specify the diagnostic criteria $[11-13,15,17,18$, $24,28,31-33,36-39,51-54,57,62,63]$, while the remaining 34 studies have clearly specified the diagnostic criteria. There were three studies $[6,36,62]$ that only defined the inclusion criteria and five that only defined the exclusion criteria $[12,22,29,34,35]$; while the remaining 40 studies did not clearly define the inclusion and exclusion criteria. There were 11 studies that adopted the random number table method $[5,10,12,18$, 19, 23, 26, 30, 36, 43, 44, 55]. Three studies used the random data method [15], the random sequence method [27], or the lottery method [28], whereas eight studies randomised according to the admission order [23, 41, $46-48,52,54,56]$. The remaining 36 studies only mentioned the term "random" in their articles. None of the studies mentioned using blinded methods. Only one study mentioned the withdrawal of a patient [16], while the remaining studies did not mention patients who became lost to follow-ups. There were in total 28 followup studies; eighteen 1-year follow-up studies [12, 15, $19,24,25,27,29,32,35,42,44,45,52,55,60-63]$, five 6 -month follow-up $[9,13,20,28,30]$, one 4month follow-up [24], one 1-month follow-up [46], two 6-week follow-up [5, 38], and one 2-week followup study [8]. All of the studies had carried out a thorough analysis on the recurrence rate. In one study that has analysed the recurrence rate [57], we failed to retrieve the recurrence rate of treatment and control groups. Another study has analysed the recurrence rate, but did not clearly stated the follow-up duration [8]. According to the Jadad scale, there were 15 studies that scored 2-points [5, 10, 12, 15, 18, 19, $23,26-28,30,36,43,44,55]$ and 35 studies that scored 1-point, while the remaining eight studies scored 0-point, indicating that these RCTs have low methodological quality (See Table 1.).

\section{Results of meta-analysis}

Outcome indicators involved in all studies included the total effective rate, recurrence rate, $\mathrm{Hp}$ eradication rate, and adverse reaction rate; of these, 50 studies have reported the total effective rate. The results of the fixed effects model combined with effect sizes showed that the difference between Jianzhong decoction and conventional WM alone was significant in treating PU (OR =4.29, 95\% CI: 3.51-5.23, $P<0.001, I^{2}=0.0 \%$, $P=0.987$; Fig. 2). Moreover, there were 24 studies reporting the $\mathrm{Hp}$ eradication rate and the results of fixed effects model combined with effect sizes showed a significant difference between Jianzhong decoction and conventional WM alone in treating PU (OR $=2.10,95 \%$ CI: 1.69-2.61, $P<0.001, I^{2}=39.6 \%, P=0.025$; Fig. 3 ). There were 24 studies reporting the recurrence rate and the results of fixed effects model combined with effect sizes showed a significant difference between Jianzhong decoction and conventional WM alone in treating PU (OR $=0.23$, 95\% CI: 0.18-0.29, $P<0.001, I^{2}=0.0 \%$, $P=0.639$; Fig. 4). There were 19 studies reporting the adverse reactions and the results of fixed effects model combined with effect sizes showed a significant difference between Jianzhong decoction and conventional WM alone in treating PU (OR $=0.20,95 \%$ CI: $0.12-0.33$, $P<0.001, I^{2}=28.1 \%, P=0.177$; Fig. 5).

\section{Sensitivity and publication bias analyses}

In order to validate the robustness of the meta-analysis, we performed the sensitivity analysis on the comparison of the total effective rate, $\mathrm{Hp}$ eradication rate, recurrence rate, and adverse reactions. The exclusion of each study individually did not significantly alter the OR values of the total effective rate, $\mathrm{Hp}$ eradication rate, recurrence rate, and adverse reactions, indicating that the metaanalysis has a relatively high result stability and reliability. The detailed results are shown in Additional file 1: Figures S1-4.

We have analysed the publication bias of the aforementioned observation indicators and the results showed that the inverted funnel plots comparing the recurrence rate, $\mathrm{Hp}$ eradication rate, and adverse reactions were basically symmetrical as shown in Figs. 6, 7, and 8, respectively. The results of Egger's test and Begg's test also confirmed the absence of significant publication bias in those included studies: $\mathrm{Hp}$ eradication rate (Egger's: $P=0.914$; Begg's: $P=0.785$ ); recurrence rate (Egger's: $P=0.213$; Begg's: $P=0.157$ ); and adverse reaction rate (Egger's: $P=0.518$; Begg's: $P=0.161$ ). In contrast, the funnel plot comparing the total effective rate was asymmetrical, indicating the possible presence of publication bias in those included studies (Fig. 9). Moreover, the results of Egger's and Begg's tests also confirmed the presence of publication bias in those included studies reporting the total effective rate (Egger's: $P=0.001$; Begg's: $P=0.202$ ). The result robustness of the meta-analysis on the total effective rate was further analysed using the trim and filling method. After the addition of 15 false-negative unpublished studies, the results showed that the point estimation and 95\% CI estimated value of the combined effect sizes did not change significantly before and after the clipping (before clipping: $\mathrm{OR}=4.06,95 \% \mathrm{CI}$ : 3.31-4.97, $n=47$; after clipping: OR $=3.29,95 \%$ CI: $2.74-3.96, n=62$ ). This indicated 


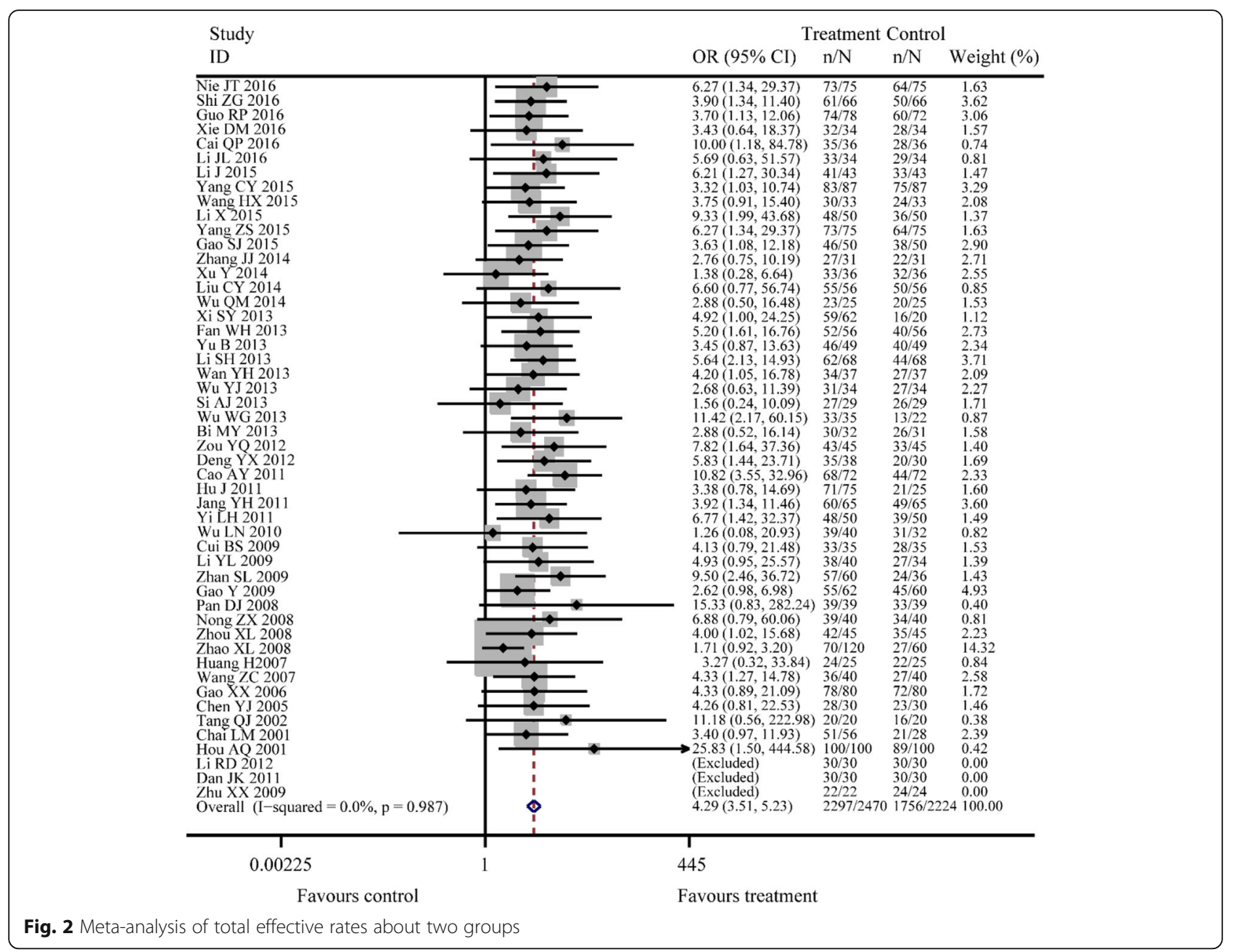

that the meta-analysis on the total effective rate has a relatively better stability.

\section{Discussion}

The PU is a result of synergistic damage caused by a gastric mucosal barrier dysfunction, enhanced gastric acid secretion, and $\mathrm{Hp}$ infection. Moreover, $\mathrm{Hp}$ infection is the main cause of PU with up to $80 \%$ and $90 \%$ of detection rate in gastric ulcers and duodenal ulcers, respectively. Therefore, an effective eradication of $\mathrm{Hp}$ has become the key to treat PU $[9,64,65]$. Presently, the triple and quadruple WM therapies are commonly used as the first-line clinical treatment program for managing PU. The WM-based therapies have the advantages of inducing rapid effects, rapid remission of clinical symptoms, high $\mathrm{Hp}$ eradication rates, and short ulcer healing times [66-68]. However, previous studies have reported several limitations of WM in treating PU, such as inducing severe side effects, increasing the patients' susceptibility to adverse reactions, poor compliance, drug resistance, recurrence, and $\mathrm{Hp}$ infection $[69,70]$. It has been also reported that patients have up to $50-70 \%$ of recurrence rates after 1 year of remission and the recurrence rates may increase up to $80-100 \%$ after 5 years [71]. Therefore, it is extremely urgent to search for a new ideal solution for the treatment of PU. The TCM Jianzhong decoction exerts various health benefits, such as "invigorate spleen and stomach", "regulate qi and resolve the phlegm", and "remove blood stasis and relieve pain". Currently, Jianzhong decoction is widely applied in the treatment of PU with a satisfactory therapeutic efficacy. Indeed, previous studies have shown that Jianzhong decoction could effectively promote the healing of ulcers and improve the Hp eradication rate in the treatment of PU with only mild side effects and low recurrence rate [10]. However, the majority of the available clinical trials only involved a small sample size and a systematic evaluation of Jianzhong decoction therapeutic efficacy is still lacking. Therefore, the clinical efficacy of Jianzhong decoction in treating PU is still controversial.

In order to elucidate this, we analysed the overall therapeutic efficacy and safety of Jianzhong decoction 


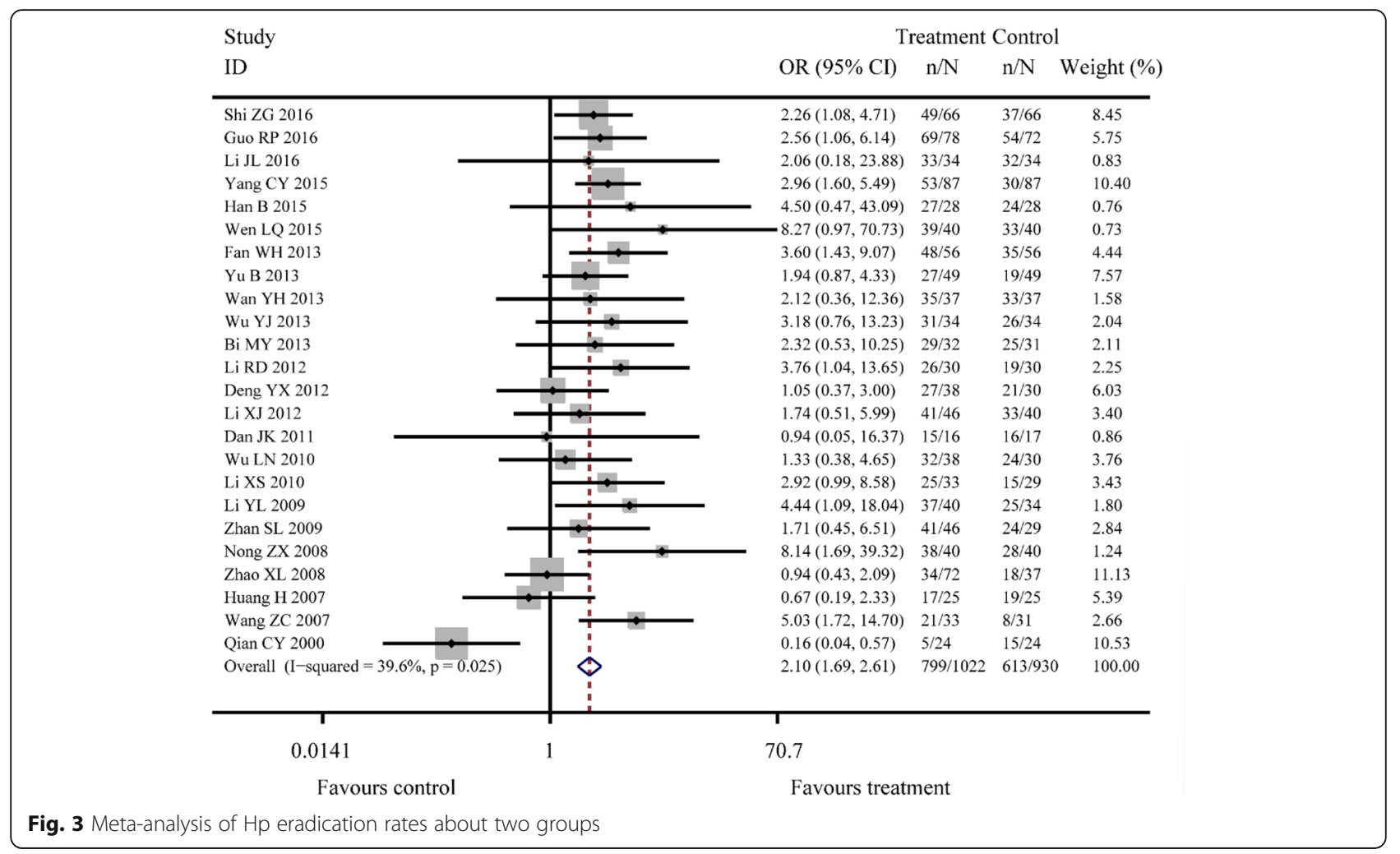

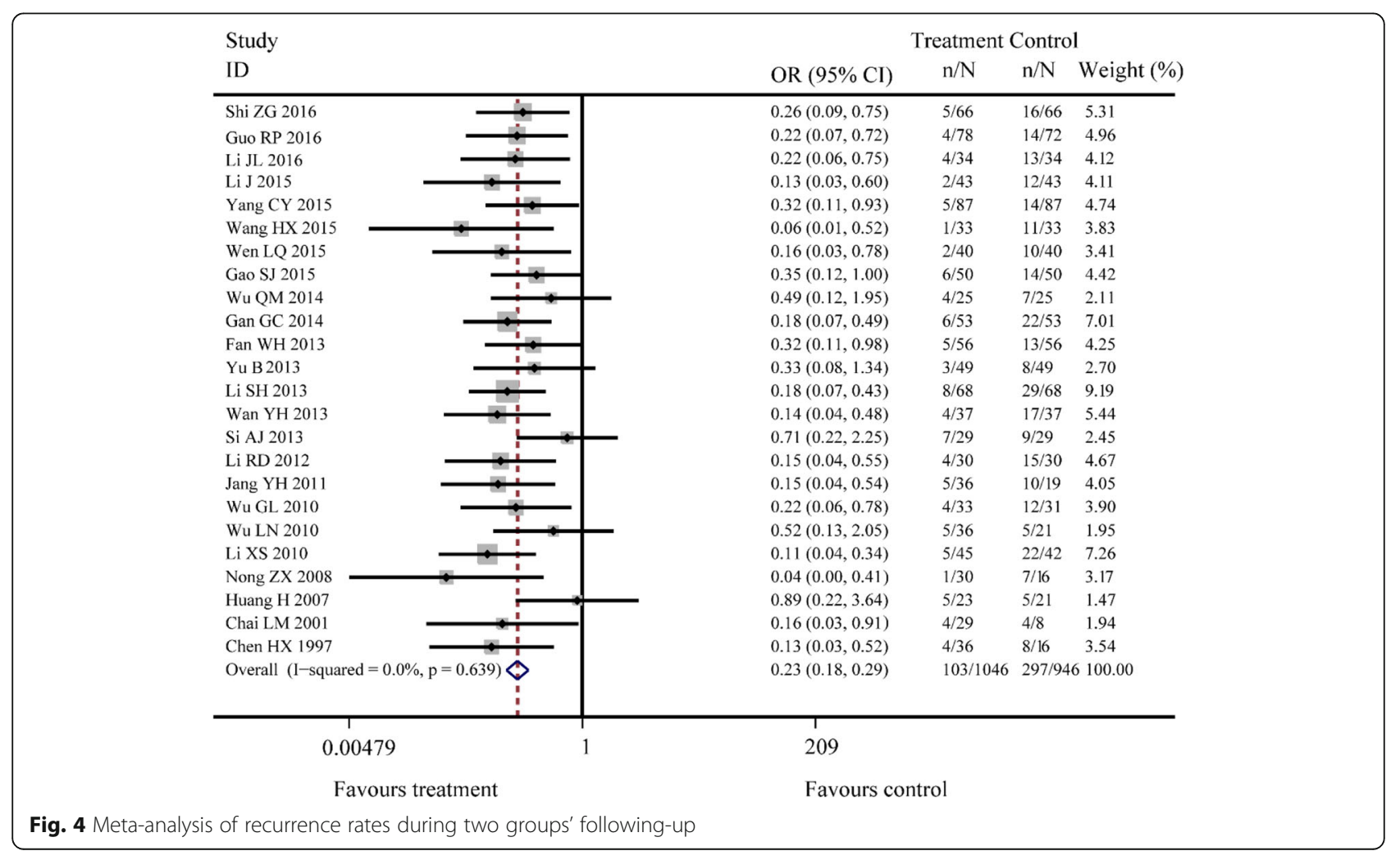




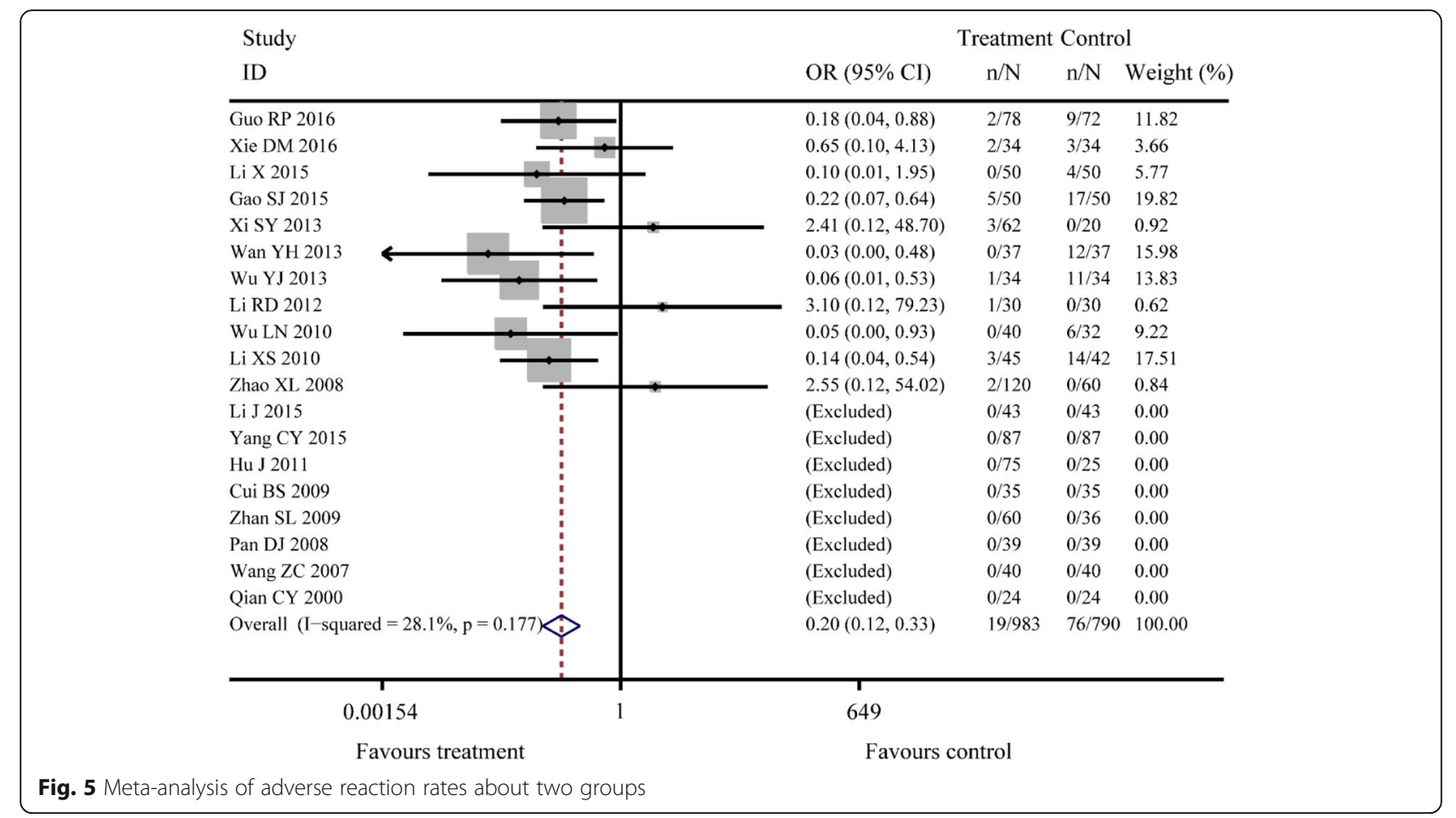

against PU using meta-analysis of systematically retrieved RCTs. We have included a total of 58 studies that included 5192 patients. Our results showed that the therapeutic efficacy of Jianzhong decoction significantly outperforms that of the conventional WM in treating PU with respect to the total effective rate, Hp eradication rate, recurrence rate, and adverse reaction rate. Our findings indicated that Jianzhong decoction has a significant clinical efficacy in treating PU with low adverse reaction and recurrence rates.

Jianzhong decoction could "warm and tonify the deficiency" and "alleviate the related pains" in PU cases. It integrates various herbs that exhibit synergistic effects

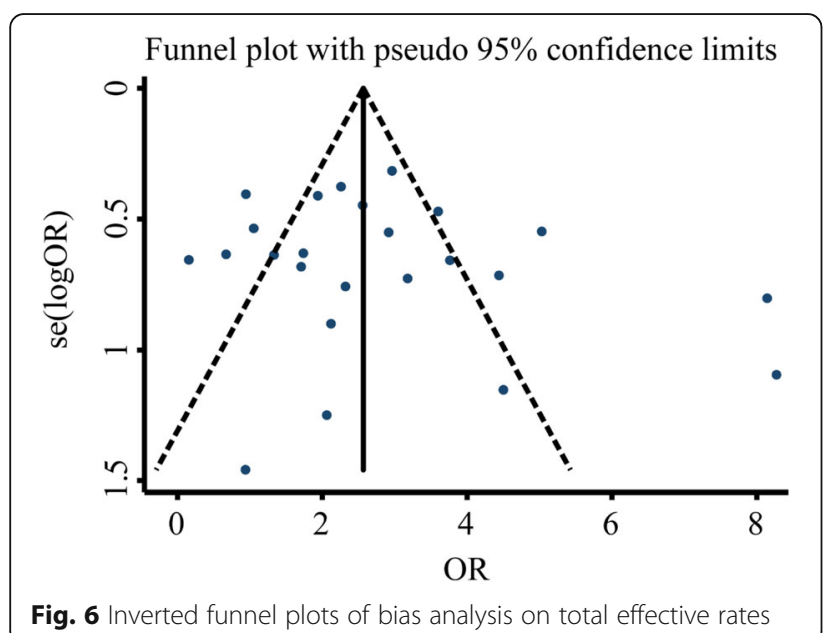

on the spleen deficiency-type PU by promoting the healing of ulcers and preventing their recurrence. In the TCM theory, middle-jiao (the middle part of a human body) is the key location for human digestion, and the pathogenesis of PU is the qi of middle-jiao hurt by coldevil. The PU, especially with pain, can be treated by Jianzhong decoction. The name, Jianzhong, means construct the qi of middle-jiao. Clinically, TCM uses some combinations of sweet and warm-natured herbs as diverse kinds of Jianzhong decoction to treat different middle-jiao's qi damage situations (such as short term or long term, slight damage or heavy damage). Here, the sweet and warm-natured herbs combinations are

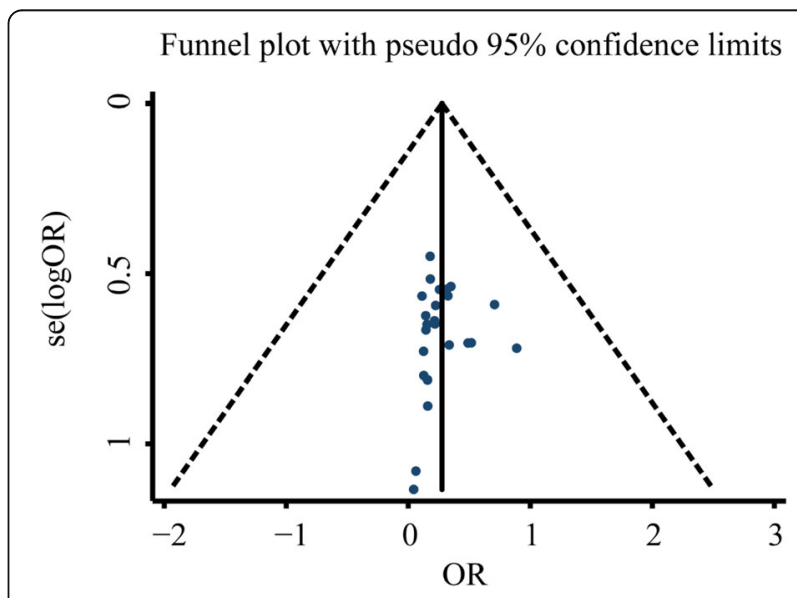

Fig. 7 Inverted funnel plots of bias analysis on Hp eradication rates 


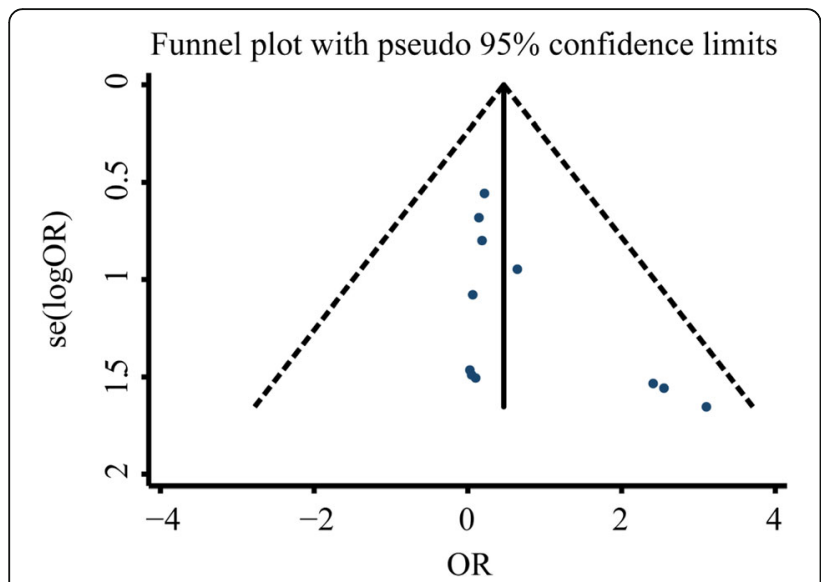

Fig. 8 Inverted funnel plots of bias analysis on recurrence rates

Sichuan pepper + Rhizoma zingiberis + Ginseng, Cassia twig + Chinese herbaceous peony + maltose, or Astragalus membranaceus + Cassia twig + Chinese herbaceous peony.

About the pharmacological aspects, these sweet and warm natured herbs can anti-inflammation, antihistamine, antibacterial, and protect gastric mucosa. Rhizoma zingiberis exerts distinct efficacy clinically. Research showed that it can induce macrophages to highly express inducible nitric oxide synthase, thereby synthesizing nitric oxide, which can mediate the activation of macrophages to influence the inflammation process [72]. Other research suggested that Ginseng polysaccharides promotes NK cell cytotoxicity in immunosuppressed mice by increasing the number of NK cells in the whole blood and upregulating the expression of perforin and granzyme. Thus, the research showed that Ginseng has a wide application prospects in the treatment of immunodeficiency diseases [73]. The major bioactive component of

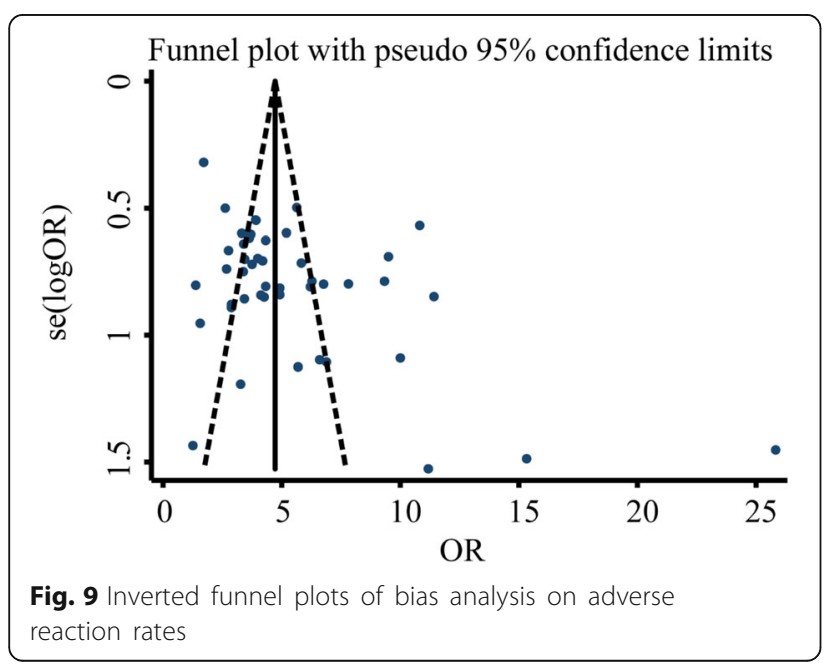

Rhizoma Zingiberis is Compound D, (E)-4-(3', 4' dimethoxyphenyl) but-3-en-1-ol, which has a strong smooth muscle relaxant, and has antihistamine and anti-inflammatory actions [74]. Ramulus Cinnamomi (Cassia twig), used to improve pharmacological effects and reduce toxicity [75]. Ginseng can inhibit H. pylori induced gastric inflammation by suppressing induction of inflammatory mediators, myeloperoxidase activity, and lipid peroxide level in $\mathrm{H}$. pyloriinfected gastric mucosa [76].

Jianzhong decoction combines various medications that not only could inhibit the secretion of gastric acid but also could enhance the expression of basic fibroblast growth factor (bFGF) in gastric mucosa of PU patients. Enhanced bFGF, thus, promotes the microvascular formation in the ulcers' granulation tissue, thereby accelerating their repair and healing [6]. Studies have shown that Jianzhong decoction has a high healing rate and level of safety, as well as a very low recurrence rate in treated PU [11]. The result of an RCT showed that, when compared with the WM control group, Jianzhong integrated with Dansen decoction has a 94.4\% of total effective rate, $88.5 \%$ of $\mathrm{Hp}$ eradication rate and $5.1 \%$ of 6 -month recurrence rate in treating PU [9]. Another study has also reported a $94.1 \%$ of total effective rate following PU treatment with Jianzhong decoction, significantly exceeding that of the control group, and in the absence of any adverse reactions [10]. In line with these previous reports, we also demonstrated that Jianzhong decoction significantly outperforms the conventional $\mathrm{WM}$ in treating $\mathrm{PU}$ with respect to the total effective rate and $\mathrm{Hp}$ eradication rate. Moreover, Jianzhong decoction resulted in significantly lower rates of recurrence and adverse reactions compared with the conventional WM. The sensitivity analysis showed that the meta-analysis results of the total effective rate, $\mathrm{Hp}$ eradication rate, recurrence rate, and adverse reaction rate are robust and reliable. However, the total effective rate showed a significant publication bias. Hence, we carried out a further analysis using the trim and filling method by supplementing with similar studies and combining their effect sizes. We found that the point estimation and 95\% CI estimated value of their combined effect sizes did not change significantly before and after the clipping, indicating that the meta-analysis results of the total effective rate are robust. The above-mentioned results suggest that Jianzhong decoction could effectively promote the healing of ulcers and alleviate the clinical symptoms in patients, in association with a high $\mathrm{Hp}$ eradication rate, low recurrence rate, and high level of safety in the treatment of PU.

Although Jianzhong decoction exhibited some benefit in PU treatment, there are a number of limitations 
to our study. Firstly, the majority of the studies had small samples. Secondly, the included studies were of low methodological quality. Finally, the majority of the included studies involved a short follow-up duration, leading to a generally low evidence for longterm using. Therefore, our observations still need to be validated by involving more high-quality, largescale RCTs in future.

\section{Conclusion}

In summary, Jianzhong decoction can improve the clinical symptoms of PU patients. So, Jianzhong decoction can be used as an ideal option for treating PU. However, quality evidence is needed to further assess its efficacy and safety.

\section{Additional file}

Additional file 1: Sensitivity analysis on the comparison of the total effective rate, $\mathrm{Hp}$ eradication rate, recurrence rate, and adverse reactions. (DOCX 808 kb)

\section{Abbreviations}

bFGF: basic fibroblast growth factor; CBMdisc: China biology medicine disc; $\mathrm{Cl}$ : Confidence interval; CNKI: China national knowledge infrastructure; CSTJ: China science and technology; Gl: Gastrointestinal; Hp: Helicobacter pylori; NSAIDs: non-steroidal anti-inflammatory drugs; OR: Odds ratio; PU: Peptic ulcer; RCTs: Randomised controlled trials; TCM: Traditional Chinese medicine; WM: Western medicine

\section{Acknowledgement}

The authors thank Dr. Xia Xu for assistance with data extraction.

\section{Funding}

N/A.

\section{Availability of data and materials}

Data are all contained within the paper.

\section{Authors' contributions}

All authors contributed to the design and concept, performed the literature searches, wrote the manuscript and critiqued the successive versions, and approved the final manuscript. YS coordinated the effort and integrated the sections and comments.

\section{Competing interests}

The authors declare no conflict of interests regarding the publication of this paper.

\section{Consent for publication \\ Not applicable.}

\section{Ethics approval and consent to participate} N/A.

\section{Publisher's Note}

Springer Nature remains neutral with regard to jurisdictional claims in published maps and institutional affiliations.

\section{Author details}

'Department of Chinese Medicine, Nankai Hospital, No. 6 Changjiang Road, Nankai District, Tianjin 300100, China. ²Department of Pharmacy, Affiliated Hospital of Logistics Institute of Chinese Armed Police Force, Tianjin 300163, China.
Received: 26 December 2016 Accepted: 4 April 2017

Published online: 14 April 2017

\section{References}

1. Moller MH, Adamsen S, Thomsen RW, Moller AM. Peptic Ulcer Perforation trial, Multicentre trial of a perioperative protocol to reduce mortality in patients with peptic ulcer perforation. Br J Surg. 2011;98(6):802-10.

2. Chu S, Wang Y. A meta-analysis of Banxiaxiexin Tang combined with omeprazole for the treatment for peptic ulcer. Res Integrated Tradit Chin West Medi. 2014;6(1):1-5.

3. Rosenstock $\mathrm{S}$, Jorgensen $\mathrm{T}$, Bonnevie $\mathrm{O}$, et al. Risk factors for peptic ulcer disease: A population Based prospective cohort study com-prising 2416 Danish adults. Gut. 2009:52(1):186-93.

4. Qian L. Application of Xiaojianzhong decoction in peptic ulcer. Guangming J Chin Med. 2008:23(7):1021-2.

5. Shi Z, Wang X. A study of the therapeutic efficacy of Xiaojianzhong decoction combined with Western medicine on spleen deficiency-type PU and its effect on serum, gastric juice EGF and gastric mucosal EGFR. World J Integrated Tradit West Med. 2016;11(5):703-6.

6. Zhu X, Ge H, Yun H. Treatment of 22 peptic ulcer patients with modified Jianzhong decoction. Traditional Chin Med Res. 2009;22(10):22-3.

7. Jadad AR, Moore RA, Carroll D. Assessing the quality of reports of randomized clinical trials: Is blinding necessary? Control Clin Trials. 1996; 17(1):1-12.

8. Nie J. Analysis of the therapeutic efficacy of Xiaojianzhong decoction and pill of Galanga and Cyperus combined with Western medicine on the spleen deficiency-type peptic ulcer. Jilin Med J. 2016;37(3):664-5.

9. Guo R. Therapy of integrated medicine in the treatment of peptic ulcer disease for 78 cases. Chin Med Mod Distance Educ China. 2016;14(12): 103-5.

10. Xie D. Study of the therapeutic efficacy of modified Xiaojianzhong decoction in patients with peptic ulcer. Cardiovasc Dis J Integrated Tradit Chin West Med. 2016:4(19):166-7.

11. Cai Q. Observation on the therapeutic efficacy of Xiaojianzhong decoction in 36 patients with spleen deficiency-type peptic ulcer. Jiankang Qianyan. 2016;23(6):161-2.

12. Li J, Fan C, Wang Q. A clinical analysis of treating peptic ulcer of Piwei Xuhan type with the Xiaojianzhong decoction plus anti-Helicobacter pylori. Clin J Chin Med. 2016:8(10):80-2.

13. Li J. Clinical analysis of Huangqi Jianzhong decoction in treating the spleen deficiency-type gastric ulcer. Shenzhen J Integrated Tradit Chin West Med. 2015;25(22):54-5.

14. Yang C. Clinical study of integrated Chinese and Western medicine in treating the spleen deficiency-type peptic ulcer. Asia Pac J Tradit Chin Med. 2015;11(6):94-5

15. Wang $\mathrm{H}$. Clinical effects observation of Huangqi Jianzhong decoction combined with Western medicine on recurrence of peptic ulcer. Clin J Chin Med. 2015:7(12):74-5.

16. Li X. A randomised parallel controlled trial of Xiaojianzhong decoction in treating the spleen deficiency-type peptic ulcer. J Pract Tradit Chin Intern Med. 2015;29(5):100-1.

17. Yang Z, You G. Analysis of the therapeutic efficacy of Xiaojianzhong and pill of Galanga and Cyperus on spleen deficiency-type peptic ulcer. Health Care Today B. 2015;11(22):218.

18. Han B. Analysis of clinical efficacy of Huangqi Jianzhong decoction on the duodenal ulcer. Contemporary Med Forum. 2015;13(3):24-5.

19. Wen L. Study of the clinical efficacy of Xiaojianzhong decoction on the spleen deficiency-type peptic ulcer. Med Inf. 2015;28(18):224-5.

20. Gao S. Clinical study of treatment of peptic ulcer with traditional Chinese medicine. Med Aesthetics Cosmetology. 2015;5(5):297.

21. Zhang J. Clinical study of Xiaojianzhong decoction and pill of Galanga and Cyperus combined with Western medicine in treating the spleen deficiency-type peptic ulcer. Contemporary Med Forum. 2014;12(2): 304-5.

22. Dong $N, X u Y$, Sun N. Clinical observation on the treatment of 36 peptic ulcer patients treated with Xiaojianzhong decoction. Nei Mongol J Tradit Chin Med. 2014;33(22):40.

23. Liu CH, Liu C. Efficacy observation of duodenal ulcer of aged people treated with Huangqi Jianzhong decoction (Traditional Chinese Medicine Formula Granules) and Rabeprazole. Liaoning J Tradit Chin Med. 2014:41(2):259-61. 
24. Wu Q. Analysis of 50 gastric ulcer patients treated with Huangqi Jianzhong decoction. Med People. 2014;27(5):138-9.

25. Gan G. Treatment of 53 peptic ulcer patients using the modified Xiaojianzhong decoction. China Health Care Nutr. 2014;2(4):2336-7.

26. Xi S, Qian L, Qian X. Treatment of 62 peptic ulcer patients with the modified Xiaojianzhong decoction. J Tradit Chin Med. 2013;54(8):703-4.

27. Fan W, He Q. Clinical observation on the treatment of spleen deficiencytype peptic ulcer with Xiaojianzhong decoction combined with AntiHelicobacter pylori. Guangming J Chin Med. 2013;28(8):1670-2.

28. Yu B. Clinical study of the treatment of spleen deficiency-type peptic ulcer with Xiaojianzhong decoction and pill of Galanga and Cyperus combined with Western medicine. Guide China Med. 2013;11(32):518-9.

29. Li S, Wei H, Huang D. Modified Xiaojianzhong decoction treating 68 cases of peptic ulcer of the spleen-stomach deficiency cold type. World Chin Med. 2013:8(11):1303-4.

30. Fu Y, Wan Y. Clinical study of the treatment of spleen deficiency-type peptic ulcer with Xiaojianzhong decoction. Contemporary Med. 2013;19(34): 155-6.

31. Wu Y. Observation on the therapeutic efficacy of traditional Chinese medicine combined with triple therapy on Helicobacter pylori-associated duodenal ulcer. Guide China Med. 2013;11(1):273-4.

32. Si A. Clinical observation on 58 cases of gastric ulcer treated with Huangqi Jianzhong decoction. China Pract Med. 2013;8(4):169.

33. Wu W. Observation on the therapeutic efficacy of Xiaojianzhong decoction on peptic ulcer. China Health Care Nutr. 2013;23(5):2723-4.

34. Bi M, Peng Q, Tong C. Clinical observation on the treatment of spleen deficiency-type peptic ulcer with Huangqi Jianzhong decoction. Hubei J Tradit Chin Med. 2013;35(2):50-1.

35. Li R, Ke J. Observation on the therapeutic efficacy of Xiaojianzhong decoction combined with Western medicine on peptic ulcer. Clin Med Eng. 2012;19(1):67-8.

36. Zou Y, Li W, Song G. Treatment of 45 cases of peptic ulcer with modified Xiaojianzhong decoction combined with Western medicine. J Bingtuan Med. 2012;32(2):24-6.

37. Deng $Y$, Wu J, Zhang A. Clinical experience of 38 cases of peptic ulcer treated with integrated Traditional Chinese medicine and Western medicine. Chin J Mod Drug Appl. 2012;6(4):94-5.

38. Li X. The comparison of healing rate and $\mathrm{Hp}$ eradication rate of duodenal ulcer between Huangqi Jianzhong decoction combined with Western medicine and Western medicine alone. Guide China Med. 2012;10(18):266-7.

39. Cao A. Observation on the therapeutic efficacy of Xiaojianzhong decoction on the peptic ulcer. Public Med Forum Mag. 2011;15(29):905-6.

40. Dan J. Effect of integrated traditional Chinese medicine and Western medicine on the expression of $\mathrm{NO}$ and IL-17 in patients with peptic ulcer. J Emerg Tradit Chin Med. 2011;20(12):1920-1.

41. Hu J, Xie C. Clinical observation on 75 cases of peptic ulcer treated with Xiaojianzhong decoction. Hunan J Tradit Chin Med. 2011;27(6):28-9.

42. Jiang $Y$. Treatment of 65 cases of peptic ulcer with integrated traditional Chinese medicine and Western medicine. China's Naturopathy. 2011;19(8):57.

43. Yi L. Observation on the therapeutic efficacy of integrated traditional Chinese medicine and Western medicine in 50 cases of peptic ulcer. Chin Foreign Health Abstr. 2011:8(18):210-1.

44. Wu G. Treatment of 45 cases of recurrent spleen deficiency-type peptic ulcer with modified Xiaojianzhong decoction. Henan Tradit Chin Med. 2010; 30(1):23-4.

45. Wu L. Clinical observation on the treatment of peptic ulcer with modified Huangqi Jianzhong decoction. Gansu Sci Technol. 2010;26(14):150-71.

46. Li X. Observation on the therapeutic efficacy of Huangqi Jianzhong decoction combined with temperature-sensitive acupuncture point paste on the peptic ulcer. Chin J Misdiagnosis. 2010;10(21):5120-1.

47. Cui B. Clinical observation on 70 cases of peptic ulcer treated with integrated traditional Chinese medicine and Western medicine. J Clin Exp Med. 2009:8(12):77-8.

48. Gao Y, Li J, Zeng Y. Observation on the therapeutic efficacy of Huangqi Jianzhong decoction combined with Western medicine in 62 cases of peptic ulcer. Jilin J Tradit Chin Med. 2009;29(6):481-2.

49. Zhan S, Tan Y. Treatment of 60 cases of peptic ulcer with modified Huangqi Jianzhong decoction. Hunan J Tradit Chin Med. 2009;25(2):57-8.

50. Li Y, Bo Y. TCM-WM treatment of 40 cases of peptic ulcer. J Pract Tradit Chin Intern Med. 2009;23(5):64-5.
51. Pan D. Clinical observation on 39 cases of peptic ulcer treated with integrated traditional Chinese medicine and Western medicine. China Pract Med. 2008;3(22):98-9.

52. Nong Z. Clinical observation on recurrence of peptic ulcer by the treatment of Huangqijianzhong soup combined with Western medicine. J Liaoning Univ Tradit Chin Med. 2008;10(7):85-6.

53. Zhou X. Clinical observation on the treatment of 45 cases of upper gastrointestinal ulcer with integrated traditional Chinese medicine and Western medicine. Forum Tradit Chin Med. 2008;23(6):39.

54. Zhao X, Lei J, Sun Z. Treatment of 120 cases of spleen deficiency-type gastric ulcer with integrated traditional Chinese medicine and Western medicine. J Shaanxi Coll Tradit Chin Med. 2008;31(5):31-2.

55. Huang $\mathrm{H}$. Clinical observation on the treatment of 25 cases of peptic ulcer with modified Xiaojianzhong decoction. Guiding J Tradit Chin Med Pharm. 2007:13(6):38-9.

56. Wang Z. A summary of 40 cases of peptic ulcer treated with Huangqi Jianzhong decoction. Hunan J Tradit Chin Med. 2007;23(4): 19-20.

57. Gao X. Treatment of 80 cases of duodenal ulcer with integrated traditional Chinese medicine and Western medicine. Shanxi J Tradit Chin Med. 2006; 22(S1):15.

58. Chen Y. Effect of modified Huangqi Jianzhong decoction on the expression of bFGF in gastric mucosa of gastric ulcer. Hunan J Tradit Chin Med. 2005; 21(6):13-4.

59. Tang Q. Treatment of 20 cases of duodenal ulcer with modified Huangqi Jianzhong decoction. Hunan J Tradit Chin Med. 2002;18(6):34.

60. Cai L. Observation on the therapeutic efficacy of Huangqi Jianzhong decoction in 56 cases of peptic ulcer. Shenzhen J Integrated Tradit Chin West Med. 2001;11(1):34-5.

61. Hou A. Treatment of 100 cases of peptic ulcer with modified Huangqi Jianzhong decoction. J Pract Tradit Chin Med. 2001;17(4):16.

62. Qian C. Observation on the therapeutic efficacy of Huangqi Jianzhong decoction and triple therapy on duodenal ulcer. Jilin J Tradit Chin Med. 2000;3(3):50.

63. Chen $\mathrm{H}$. Investigation of the treatment of duodenal ulcer with omeprazole combined with Huangqi Jianzhong decoction. Chin J Integrated Tradit West Med Digestion. 1997;5(3):177.

64. Farzaei $\mathrm{MH}$, Abdollahi M, Rahimi R. Role of dietary polyphenols in the management of peptic ulcer. World J Gastroenterol. 2015;21(21): 6499-517.

65. Farzaei MH, Rahimi R, Abdollahi M, Abbasabadi Z. An Evidence-based Review on Medicinal Plants used for the Treatment of Peptic Ulcer in Traditional Iranian Medicine. Int J Pharmacol. 2013;9(2):108-24.

66. Berrutti M, Astegiano M, Smedile A, et al. Efficacy of amoxicillin plus clavulanic acid-based triple therapy for Helicobacter pylori eradication: A retrospective study. Pan Minerva Med. 2010;52(4):361-2.

67. Lin LC, Hsu TH, Huang KW, Tam KW. Nonbismuth concomitant quadruple therapy for Helicobacter pylori eradication in Chinese regions: A metaanalysis of randomized controlled trials. World J Gastroenterol. 2016;22(23): 5445-53.

68. Gisbert JP, Pajares JM. Systematic review and meta-analysis: is 1-week proton pump inhibitor-based triple therapy sufficient to heal peptic ulcer? Aliment Pharmacol Ther. 2005;21(7):795-804

69. Huang CY, Lai WY, Sun MF, et al. Prescription patterns of traditional Chinese medicine for peptic ulcer disease in Taiwan: A nationwide population-based study. J Ethnopharmacol. 2015;12(176):311-120.

70. Cohen S, Bueno de Mesquita M, Mimouni FB. Adverse effects reported in the use of gastroesophageal reflux disease treatments in children: a 10 years literature review. Br J Clin Pharmacol. 2015;80(2): 200-8.

71. Khoshbaten M, Ghaffarifar S, Jabbar Iman A, et al. Effects of early oral feeding on relapse and symptoms of upper gastrointestinal bleeding in peptic ulcer disease. Dig Endosc. 2013;25(2):125-9.

72. Liao H. Effects of Shengjiang (Zingiberis Rhizoma Recens) and Its Processed Products on Nitric Oxide Production in Macrophage RAW 264.7 Cells. Evid Based Complement Alternat Med. 2015;2015:828156.

73. Sun Y, Guo M, Feng Y, Zheng H, Lei P, Ma X, Han X, Guan H, Hou D. Effect of ginseng polysaccharides on NK cell cytotoxicity in immunosuppressed mice. Exp Ther Med. 2016;12(6):3773-7.

74. Khemawoot $\mathrm{P}$, Hunsakunachai $\mathrm{N}$, Anukunwithaya T, Bangphumi $\mathrm{K}$, Ongpipattanakul B, Jiratchariyakul W, Soawakontha R, Inthachart T, 
Dechatiwongse Na Ayudhya T, Koontongkaew S, Poachanukoon O. Pharmacokinetics of Compound D, the Major Bioactive Component of Zingiber cassumunar. Rats Planta Med. 2016;82(13):1186-91.

75. Wei P, Huo HL, Ma QH, Li HC, Xing XF, Tan XM, Luo JB. Pharmacokinetic comparisons of five ephedrine alkaloids following oral administration of four different Mahuang-Guizhi herb-pair aqueous extracts ratios in rats. J Ethnopharmacol. 2014;155(1):642-8.

76. Bae M, Jang S, Lim JW, Kang J, Bak EJ, Cha JH, Kim H. Protective effect of Korean Red Ginseng extract against Helicobacter pylori-induced gastric inflammation in Mongolian gerbils. J Ginseng Res. 2014;38(1):8-15.

Submit your next manuscript to BioMed Central and we will help you at every step:

- We accept pre-submission inquiries

- Our selector tool helps you to find the most relevant journal

- We provide round the clock customer support

- Convenient online submission

- Thorough peer review

- Inclusion in PubMed and all major indexing services

- Maximum visibility for your research

Submit your manuscript at www.biomedcentral.com/submit
Biomed Central 